This is the peer reviewed version of the following article: Horváth, E., et al., The role of Arabidopsis glutathione transferase F9 gene under oxidative stress in seedlings, Acta Biologica Hungarica 66(4): 406-418 (2015), which has been published in final form at http://dx.doi.org/10.1556/018.66.2015.4.5.

\title{
The role of Arabidopsis glutathione transferase F9 gene under oxidative stress in seedlings
}

Edit Horváth ${ }^{1}$, Krisztina Bela ${ }^{1}$, Csaba Papdi $^{2}$, Ágnes Gallé ${ }^{1}$, László Szabados ${ }^{2}$, Irma Tari ${ }^{1}$, Jolán Csiszár ${ }^{1}$

${ }^{1}$ Department of Plant Biology, Faculty of Sciences, University of Szeged, Közép fasor 52., H6726 Szeged, Hungary

${ }^{2}$ Institute of Plant Biology, Biological Research Centre of HAS, Temesvári krt. 62., H-6726 Szeged, Hungary

Corresponding author:

Edit Horváth

E-mail: horvathedo@yahoo.com

Running title: The role of AtGSTF9 in oxidative stress response 
Abstract

Arabidopsis thaliana contains 54 soluble glutathione transferases (GSTs, EC 2.5.1.18), which are thought to play major roles in oxidative stress responses, but little is known about the function of individual isoenzymes. The role of AtGST phi 9 (GSTF9) in the salt- and salicylic acid response was investigated using 2-week-old Atgstf9 and wild type (Wt) plants. Atgstf9 mutants accumulated more ascorbic acid (AsA) and glutathione (GSH) and had decreased glutathione peroxidase (GPOX) activity under control conditions. Treatment of 2-week-old seedlings with $10^{-7} \mathrm{M}$ salicylic acid (SA) for $48 \mathrm{~h}$ resulted in elevated $\mathrm{H}_{2} \mathrm{O}_{2}$ level and enhanced GST activity in Atgstf9 plants, $10^{-5} \mathrm{M}$ SA treatment enhanced the malondialdehyde and dehydroascorbate contents compared to Wt. 50 and $150 \mathrm{mM} \mathrm{NaCl}$ increased the GST activity, AsA and GSH accumulation in Atgstf9 seedlings more pronounced than in Wt plants. We found that the Atgstf9 mutants had altered redox homeostasis under control and stress conditions, in which elevated AsA and GSH levels and modified GST and GPOX activities may play significant role. The halfcell potential values calculated from the concentration of GSH and GSSG indicate that this GST isoenzyme has an important role in the salt stress response.

Key words: Arabidopsis thaliana; ascorbic acid; glutathione; glutathione transferase phi 9; glutathione peroxidase activity

\section{INTRODUCTION}

Plant glutathione transferases (GSTs, EC 2.5.1.18) are ubiquitous enzymes that catalyze the conjugation of the tripeptide glutathione (GSH; $\gamma$-Glu-Cys-Gly) to an electrophilic centre within 
a small acceptor molecule. A key role of GSTs is their ability to inactivate toxic compounds and this is crucial to the survival of cells and organisms both under normal and different stress conditions [13]. All plants possess multiple cytosolic GSTs, each of which displays distinct catalytic as well as non-catalytic binding properties [11]. Arabidopsis thaliana contains 54 soluble GSTs which can be divided into seven distinct classes: tau, phi, theta, zeta, lambda, dehydroascorbate reductase (DHAR) and tetrachlorohydroquinone dehalogenase (TCHQD). Plant GSTs that belong to tau, phi and theta classes exhibit GSH-dependent peroxidase activity (GPOX, EC 1.11.1.9), reducing organic hydroperoxides, e.g. lipid peroxides, to monohydroxyalcohols. The phi GSTs (GSTFs) represent a large, plant-specific class of proteins with 13 members in Arabidopsis that are likely to be involved in conferring tolerance to oxidative stress [4]. Increased expression of phi class GSTs was provoked by ethylene, methyl jasmonate, $\mathrm{H}_{2} \mathrm{O}_{2}$ and salicylic acid (SA) treatments [28]. However, very little is known about the function of individual GSTFs because of their possible functional redundancy. AtGSTF9 (At2g30860) is a highly abundant and constitutively expressed gene [28], which can be readily detected at the protein level [21]. Several GSTF enzymes (GSTs F6, F7, F9 and F10) were knocked down using RNAi which did not lead to phenotypic alterations, suggesting that individual GSTFs are non-essential for normal growth [22]. However, overexpression of AtGSTF9 conferred dominant, estradiol-dependent salt tolerance to transgenic Arabidopsis [18].

Salt stress is one of the most important stress factors which negatively affect plant growth and development. The plant response to salinity can be separated into two phases that are characterized as osmotic and ionic stress [17], but as a secondary phenomenon most stress factors are usually associated with oxidative burst, leading to oxidative stress [19]. Salt stress 
leads to the accumulation of reactive oxygen species (ROS) such as singlet oxygen $\left({ }^{1} \mathrm{O}_{2}\right)$, superoxide radical $\left(\mathrm{O}_{2}{ }^{--}\right)$, hydroxyl radical $\left(\mathrm{OH}^{*}\right)$ and hydrogen peroxide $\left(\mathrm{H}_{2} \mathrm{O}_{2}\right)$ [17].

ROS are highly reactive and cytotoxic compounds in cells, therefore a tight control is needed to balance the individual ROS and reactive nitrogen species, ROS-producing enzymes, antioxidants and their oxidation/reduction states [5, 2]. All these contribute to the general redox homeostasis, which plays a central role in the mediation of plant responses to abiotic or biotic stresses [20]. Salicylic acid (SA) is a plant hormone with well-known effect on ROS-accumulation and influence on redox status of cells through regulation of ROS generating enzymes. ROS triggered changes have central role in GSH/GSSG and thiol-disulphide exchanges of non-expressor of PR protein 1 (NPR1) and in regulation the expression of pathogenesis-related (PR) proteins [16]. Monomerization of NPR1, changes in NPR1 thiol-disulphide status and ROS-induced increases in cytosolic calcium, which cause activation of kinase cascades are all important features of oxidative signalling [6].

Our experimental system was designed to investigate the particular role of AtGSTF9 in oxidative stress responses induced by $\mathrm{NaCl}$ or salicylic acid and we measured GST and GPOX activities, the $\mathrm{H}_{2} \mathrm{O}_{2}$ and malondialdehyde (MDA) contents, and the ascorbate and glutathione pool in the seedlings. The aim of our study was to gain deeper insights into the mechanisms which may be influenced by AtGSTF9 using a T-DNA insertion mutant. After $48 \mathrm{~h}$ salt- or SA treatments, Atgstf9 mutants had elevated ascorbic acid (AsA) and GSH contents assuming that these plants had a modified redox homeostasis. Our results indicate that AtGSTF9 plays a role in the fine tuning of redox homeostasis under control and stress conditions.

\section{MATERIALS AND METHODS}


Plant material and growth conditions

14-day-old wild type (Col-0) and Atgstf9 mutant Arabidopsis thaliana L. seedlings were used in all experiments. The knockout Arabidopsis line (SALK_148672C) containing a T-DNA insertion in GSTF9 (At2g30860) gene was obtained from the Salk Institute (http://signal.salk.edu/cgibin/tdnaexpress). The relative transcript amount of AtGSTF9 was $2^{-\Delta \Delta C t}=0.065$ in the Atgstf9 compared to the wild type under control conditions. Stress treatments were carried out on twoweek-old in vitro grown plants by placing them onto agar-solidified culture medium supplemented with 50 and $150 \mathrm{mM} \mathrm{NaCl}$ or $10^{-7}$ and $10^{-5} \mathrm{M}$ salicylic acid (SA). The Petri dishes were kept in growth chamber (Fitoclima S 600 PLH, Aralab, Portugal) at a photon flux density of $100 \mu \mathrm{mol} \mathrm{m} \mathrm{s}^{-1}$ (10/14 day/night period), at a relative humidity of $70 \%$ and $21^{\circ} \mathrm{C}$. Samples were taken after $48 \mathrm{~h} \mathrm{NaCl}$ or SA treatments. The experiments were repeated at least two times, the measurements were performed in three replicates unless indicated otherwise.

\section{Investigation of viability using fluorescent microscopy}

Fluorescein diacetate (FDA) was applied for the determination of cell viability according to Lehotai et al. [12]. Fluorescence intensity values were determined in $80 \mu \mathrm{m}$ diameter circles, at $150 \mu \mathrm{m}$ distance from the root tip in roots and $600 \mu \mathrm{m}$ diameter circles in leaves by Zeiss Axiowert 200M microscope (Carl Zeiss, Jena, Germany) using Axiovision Rel. 4.8 software. The diameter of circles was not modified during the experiments. The measurements were performed in 10 replicates, mean $\pm \mathrm{SE}$ are given on the figures.

Determination of $\mathrm{H}_{2} \mathrm{O}_{2}$ level 
Hydrogen peroxide level was measured spectrophotometrically after homogenization of $200 \mathrm{mg}$ shoot or root tissue on ice with $300 \mu 10.1 \%$ trichloroacetic acid (TCA) as described earlier [8]. The amount of $\mathrm{H}_{2} \mathrm{O}_{2}$ was calculated using a standard curve prepared with 0.1-5 $\mu \mathrm{mol} \mathrm{ml}{ }^{-1} \mathrm{H}_{2} \mathrm{O}_{2}$ concentrations.

\section{Malondialdehyde determination}

Malondialdehyde (MDA) formation was followed by using the thiobarbituric acid method [1]. 50 mg tissue was homogenized with $500 \mu 10.1 \%$ TCA and $50 \mu 14 \%$ butylhydroxytoluene was added to avoid further lipid peroxidation. The absorbance was measured at $532 \mathrm{~nm}$ and adjusted for nonspecific absorbance at $600 \mathrm{~nm}$. MDA concentration was calculated using an extinction coefficient of $155 \mathrm{mM}^{-1} \mathrm{~cm}^{-1}$.

\section{Determination of glutathione transferase and glutathione peroxidase enzyme activities}

Glutathione transferase (GST, EC 2.5.1.18) activity was determined as published earlier [1] with some modifications. $200 \mathrm{mg}$ tissue was homogenized on ice in $1 \mathrm{ml} 100 \mathrm{mM}$ phosphate buffer (pH 7.0) containing $1 \mathrm{mM}$ phenylmethylsulfonyl fluoride and $1 \%$ polyvinyl-polypirrolidone. The homogenate was centrifuged for $20 \mathrm{~min}$ at $10000 \mathrm{~g}$ at $4{ }^{\circ} \mathrm{C}$ and the supernatant was used for enzyme activity assays. GST activity was determined spectrophotometrically by using an artificial substrate, 1-chloro-2,4-dinitrobenzene (CDNB, Sigma-Aldrich). GST activity was given in nkat ( $\mathrm{nmol}$ conjugated products $\mathrm{sec}^{-1}, \varepsilon_{340}=9.6 \mathrm{mM}^{-1} \mathrm{~cm}^{-1}$ ). Glutathione peroxidase (GPOX, EC 1.11.1.9) activity was measured with cumene hydroperoxide (CHP; Sigma-Aldrich) as a substrate. The reaction mixture contained $4 \mathrm{mmol} \mathrm{l}^{-1} \mathrm{GSH}, 0.2 \mathrm{mmol}^{-1} \mathrm{NADPH}, 0.05 \mathrm{U}$ of GR (from baker's yeast, Sigma-Aldrich), $100 \mu \mathrm{l}$ enzyme extract, and $0.5 \mathrm{mmol}^{-1}$ substrate in 
phosphate buffer $\left(0.1 \mathrm{~mol} \mathrm{l}^{-1}, \mathrm{pH} 7.0\right)$ in a total volume of $1 \mathrm{ml}$. GPOX activity was given in nkat ( $\mathrm{nmol}$ of converted NADPH sec ${ }^{-1}, \varepsilon_{340}=6.22 \mathrm{mM}^{-1} \mathrm{~cm}^{-1}$ ).

\section{Ascorbate and glutathione extraction and determination}

Ascorbate and glutathione contents were determined according to Tari et al. [26]. Two-hundred mg of seedlings was homogenized with $0.8 \mathrm{ml}$ of $5 \%$ TCA. The homogenate was centrifuged at $10000 \mathrm{~g}$ for $20 \mathrm{~min}$ at $4^{\circ} \mathrm{C}$ and the supernatant was used for further determinations. To assay total ascorbate (AsA), $100 \mu \mathrm{l}$ of $10 \mathrm{mM}$ dithiothreitol (DTT) was added to the extract and the excess of DTT was removed by adding $100 \mu \mathrm{l}, 0.5 \%$ (w:v) N-ethylmaleimid (NEM). Ascorbate concentrations were determined spectrophotometrically at $525 \mathrm{~nm}$. Dehydroascorbate (DHA) content was calculated as the difference between the concentration of total and reduced ascorbate.

Total and oxidized glutathione concentrations were measured spectrophotometrically using an enzymatic assay. To mask reduced glutathione (GSH) 4-vinylpyridine was added to the extract and incubated for $60 \mathrm{~min}$. The reaction mixture contained $0.1 \mathrm{M}$ phosphate buffer $\mathrm{pH} 7.5,1 \mathrm{mM}$ 5,5'-dithiobis(2-nitrobenzoic acid) (DTNB), $1 \mathrm{mM} \mathrm{NADPH,} 1 \mathrm{U}$ of glutathione reductase (GR baker's yeast, Sigma) and $20 \mu \mathrm{l}$ of the tissue extract in $1 \mathrm{ml}$ volume. GSH content was calculated from the difference between the concentration of total and oxidized glutathione. Standard curves were obtained for total glutathione and GSSG within the $0-2 \mu \mathrm{M}$ range.

The reduction potential of the GSH/GSSG couple was determined with the Nernst equation using the form of Schafer and Buettner $[23]: \mathrm{E}_{\mathrm{hc}}=-240-(59.1 / 2) \log \left([\mathrm{GSH}]^{2} /[\mathrm{GSSG}]\right) \mathrm{mV}$; where $\mathrm{E}_{\mathrm{hc}}$ $=E_{\mathrm{GSSG} / 2 \mathrm{GSH}}$ : the half-cell redox potential of glutathione, $-240 \mathrm{mV}$ : the standard reduction potential of glutathione on $25^{\circ} \mathrm{C}, \mathrm{pH}=7.0$. 


\section{Statistical analysis}

Statistical analysis was carried out with SigmaPlot 11.0 software (SigmaPlot, Milano, Italy) by Duncan's test and differences were considered significant at $\mathrm{P} \leq 0.05$. Data presented here are the means \pm SD of at least 3 measurements unless indicated otherwise.

\section{RESULTS}

\section{Different viability of wild type and Atgstf9 mutant plants under adverse conditions}

The root lengths of 2-week-old Atgstf9 seedlings was significantly decreased compared to wild type plants, but only slight differences were detected between the plants in the presence of 150 $\mathrm{mM} \mathrm{NaCl}$ or $10^{-5} \mathrm{M}$ (data not shown). The viability of wild type and Atgstf9 mutant Arabidopsis was similar under control conditions. Treatment with 50 and $150 \mathrm{mM} \mathrm{NaCl}$ decreased the viability of wild type Arabidopsis leaves by $50 \%$ and $60 \%$, while viability of Atgstf9 mutants was reduced by $70 \%$ and $80 \%$, respectively. Similar tendencies could be observed in roots of wild type and Atgstf9 plants (Fig. 1). SA treatment decreased the viability of wild type seedlings only in leaves, but $10^{-7} \mathrm{M} \mathrm{SA}$ did not affected it in mutants (Fig. 1).

\section{$\mathrm{H}_{2} \mathrm{O}_{2}$ accumulation and oxidative damage in $\mathrm{SA}$ - and salt-treated plants}

$\mathrm{H}_{2} \mathrm{O}_{2}$ and MDA accumulation during the stress was detected using the whole seedlings. Under control conditions, the wild type and the mutant seedlings had similar $\mathrm{H}_{2} \mathrm{O}_{2}$ and MDA contents. Salt treatment induced $\mathrm{H}_{2} \mathrm{O}_{2}$ accumulation only in Atgstf9 mutants when it was applied in 150 $\mathrm{mM}$ concentration but the MDA content was elevated both in wild type and mutant seedlings. $10^{-}$ 
${ }^{7} \mathrm{M}$ SA triggered the most pronounced increase in the $\mathrm{H}_{2} \mathrm{O}_{2}$ content and the MDA level was also increased compared to the untreated plants (Fig. 2).

Glutathione transferase and glutathione peroxidase activity in wild type and mutant plants $150 \mathrm{mM} \mathrm{NaCl}$ or $10^{-5} \mathrm{M} \mathrm{SA}$ treatment increased the glutathione transferase activity in the wild type plants. The mutant seedlings had significantly higher GST activity after 50 or $150 \mathrm{mM} \mathrm{NaCl}$ treatment or after application of $10^{-7} \mathrm{M} \mathrm{SA}$ comparing to the wild type. GST activity in $10^{-5} \mathrm{M}$ SA-treated Atgstf9 plants declined compared to other treatments (Fig. 3). The GPOX activity was significantly reduced in Atgstf9 mutant seedlings under control conditions. Salt treatment reduced the GPOX activity both in the wild type and in mutant plants, however in Atgstf9 it was lower than in the wild type. SA elevated the GPOX activity both in wild type and mutant plants, and there were no significant difference between them after $48 \mathrm{~h}$ of treatments (Fig. 3).

\section{Role of the ascorbate-glutathione pool in the $\mathrm{NaCl}$ and SA stress responses}

The total ascorbate and glutathione contents of the Atgstf9 seedling were higher compared to the wild type under control conditions. Salt treatment increased the total ascorbate content of seedlings after two days, and the Atgstf9 Arabidopsis accumulated more ascorbate. The proportion of the oxidised form of ascorbate (DHA), was not changed after $50 \mathrm{mM} \mathrm{NaCl}$, but was higher in plants treated with $150 \mathrm{mM} \mathrm{NaCl}$, where the glutathione accumulation was also induced, and this accumulation was more pronounced in Atgstf9 plants. SA treatments dramatically reduced AsA, while enhanced the DHA contents in both genotypes, and increased the accumulation of GSH in wild type and in Atgstf9 plants. The amount of GSSG barely changed in these treatments and was similar in wild type and mutant plants (Fig 4). The used salt 
treatments resulted in distinct $E_{G S S G / 2 G S H}$ levels, because the wild type and Atgstf9 plants had different GSH and GSSG contents (Table 1).

\section{DISCUSSION}

The primary components of the system to combat excess ROS in plants are non-enzymatic antioxidants (such as ascorbate, glutathione, carotenoids, tocopherols) and enzymes which are involved in quenching of reactive oxygen species (superoxide dismutase, catalase, peroxidases), re-reduction of the members of ascorbate-glutathione cycle and/or in maintenance of cellular redox state (such as ascorbate peroxidase, glutathione reductase) and other proteins with general or specific roles in stress responses, e.g. GSTs [15, 9, 20]. Sappl et al. [21] reported that AtGSTF9 among a set of other GSTs (AtGSTF2, AtGSTF6/7, AtGSTF10, AtDHAR1, AtDHAR3, AtGSTU5 and AtGSTU19) is common to non-treated shoots and cell culture [21].

Despite the high abundance, the decreased expression of ATGSTF9 did not affect the GST activity measured with CDNB substrate in the mutants under control conditions. After applying $\mathrm{NaCl}$ or SA the total extractable GST activity was even higher supposedly because of activation of other GST isoenzymes and/or GST coding sequences. However, specific GPOX activity data revealed that AtGSTF9 has important role both under control and salt stress conditions of plants (Fig. 3). While the viability, level of $\mathrm{H}_{2} \mathrm{O}_{2}$ or the lipid peroxidation marker MDA showed similar values in the wild type and mutant seedlings under control conditions, the level of AsA and GSH was increased in the mutants. Ascorbate and glutathione are non-enzymatic antioxidants which may play roles in signal transduction and/or transmission during cell death and defence responses. Moreover, ascorbate was suggested to be the redox molecule which primarily 
regulates plant development, and glutathione is also involved in this process, although it is mainly important for stress defence and signalling [20]. In accordance with these, in our experiments the role of increased AsA and GSH pools was more pronounced after applying $\mathrm{NaCl}$ or SA treatments for two days on the 2-week-old plants. While the salt stress affected mainly the size of the AsA pool, SA resulted in shift of AsA pool towards the DHA. Detoxification of $\mathrm{H}_{2} \mathrm{O}_{2}$ through the glutathione - ascorbate cycle leads to a transient change in the degree of oxidation of the cellular glutathione pool, and thus a change in the glutathione redox potential, which is not only an indicator of the general redox status, but it is part of the redox regulatory mechanism [14, 10, 24]. Changes in the concentration and in the reduction potential of the GSH/GSSG and other redox couples make possible the fine regulation of the cellular redox environment and consequently the growth of the plants.

Glutathione has pivotal role in oxidative stress defence, and depletion of the cytosolic GSH pool is associated with large changes in the abundance of transcripts encoding proteins that are involved in oxidative defence [7]. Mining of the proteome data for GSH-associated genes showed that disruption of the pathway for the synthesis and degradation of glutathione in the Atggt1 knockout leaves was associated with the induction of genes encoding four GSTs in the phi class (GSTF2, GSTF6, GSTF9, and GSTF10), a GSH peroxidase (GPX1), and glyoxylase II [27]. The important role of the a high GSH/GSSG ratio, maintained by increased GSH synthesis and/or GSSG reduction, in the efficient protection of plants against abiotic stress-induced accumulation of ROS was indicated at several plant species [25].

Based on our findings, the elevated AsA and GSH content of Atgstf9 mutants may be the result of a complex defence mechanism which helps to maintain the cellular homeostasis in the case of very low AtGSTF9 expression. These results suggest a modified redox homeostasis which could 
induce a redox signalling pathway resulting in even elevated GST activities under stress conditions.

\section{CONCLUSION}

The observed differences, induced by salt stress and SA in the viability, GPOX activity, AsA and GSH contents between Atgstf9 and Col-Wt plants can be in connection with the different redox status measured in the seedlings. In our experiments, the $\mathrm{E}_{h c}$ values calculated from the concentration of GSH and GSSG indicate that AtGSTF9 isoenzyme has a positive role in the salt stress response of seedlings by modulating the short-term redox homeostasis of plants. The mutants had significantly lower GPOX activity than the wild type, however, we did not found a significant decrease in GST activity. This result can be explained by the high redundancy of GSTs, supposedly other isoenzymes were induced. The results of the present study clearly show that the lack of AtGSTF9 increased the AsA and GSH pools, affected the redox status, which might influence the long-term growth of plants.

\section{ACKNOWLEDGEMENT}

This work was supported by the Hungarian National Scientific Research Foundation [grant number OTKA K 105956], Hungary-Serbia IPA Cross-border Co-operation Programme (HUSRB/1203/221/173) and by the European Union and the Sate of Hungary, co-financed by the European Social Fund in the framework of TÁMOP 4.2.4.A/2-11/1-2012-0001 'National Excellence Program' scholarship to E. H. and K. B. 


\section{REFERENCES}

1. Csiszár, J., Szabó, M., Erdei, L., Márton, L., Horváth, F., Tari, I. (2004) Auxin autotrophic tobacco callus tissues resist oxidative stress: the importance of glutathione S-transferase and glutathione peroxidase activities in auxin heterotrophic and autotrophic calli. J. Plant Physiol. $161,691-699$.

2. De Tullio, M. C. (2010) Antioxidants and redox regulation: Changing notions in a changing world. Plant Physiol. Bioch. 48, 289-291.

3. Després, C., Chubak, C., Rochon, A., Clark, R., Bethune, T., Desveaux, D., Fobert, P. R. (2003) The Arabidopsis NPR1 disease resistance protein is a novel cofactor that confers redox regulation of DNA binding activity to the basis domain/leucine zipper transcription factor TGA1. Plant Cell 15, 2181-2191.

4. Dixon, D. P., Edwards, R. (2010) Glutathione transferases. Arabidopsis Book 8, e0131.

5. Foyer, C. H., Noctor, G. (2005) Redox homeostasis and antioxidant signaling: A metabolic interface between stress perception and physiological responses, Plant Cell 17, 1866-1875.

6. Foyer, C. H., Noctor, G. (2005) Oxidant and antioxidant signalling in plants: a re-evaluation of the concept of oxidative stress in a physiological context. Plant Cell Environ. 28, 10561071.

7. Foyer, C. H., Noctor, G. (2011) Ascorbate and glutathione: the heart of the redox hub. Plant Physiol. 155, 2-18. 
8. Gémes, K., Poór, P., Horváth, E., Kolbert, Z., Szopkó, D., Szepesi, Á., Tari, I. (2011) Crosstalk between salicylic acid and $\mathrm{NaCl}$-generated reactive oxygen species and nitric oxide in tomato during acclimation to high salinity. Physiol. Plantarum 142, 179-192.

9. Jaleel, C. A., Riadh, K., Gopi, R., Manivannan, P., Ines, J., Al-Juburi, H., Chang-Xing, Z., Hong-Bo, S., Panneerselvam, R. (2009) Antioxidant defense responses: physiological plasticity in higher plants under abiotic constraints. Acta Physiol. Plant. 31, 427-436.

10. Kocsy, G., Tari, I., Vanková, R., Zechmann, B., Gulyás, Z., Poór, P., Galiba, G. (2013) Redox control of plant growth and development. Plant Sci. 211, 77-91.

11. Labrou, N. E., Papageorgiou, A. C., Pavli, O., Flemetakis, E. (2015) Plant GSTome: structure and functional role in xenome network and plant stress response. Curr. Opin. Biotech. 32, $186-194$.

12. Lehotai, N., Pető, A., Bajkán, Sz., Erdei, L., Tari, I., Kolbert Zs. (2011) In vivo and in situ visualization of early physiological events induced by heavy metals in pea root meristem. Acta Physiol. Plant. 33, 2199-2207.

13. Marrs, K.A. (1996) The functions and regulation of glutathione S-transferases in plants. Annu. Rev. Plant Phys. 47, 127-158.

14. Meyer, A. J. (2008). The integration of glutathione homeostasis and redox signaling. J. Plant Physiol. 165, 1390-1403.

15. Mittler, R. (2002) Oxidative stress, antioxidants and stress tolerance. Trends Plant Sci. 7, $405-410$.

16. Mou, Z., Fan, W., Dong, X. (2003) Inducers of plant systemic acquired resistance regulate NPR1 function through redox changes. Cell 27, 935-944. 
17. Munns, R., Tester, M. (2008) Mechanisms of salinity tolerance. Annu. Rev. Plant Biol. 59, $651-681$.

18. Papdi, C., Ábrahám, E., Joseph, M. P., Popescu, C., Koncz, C., Szabados, L. (2008) Functional identification of Arabidopsis stress regulatory genes using the controlled cDNA overexpression system. Plant Physiol. 147, 528-542.

19. Pál, M., Szalai, G., Kovács, V., Gondor, O. K., Janda, T. (2013) Salicylic acid-mediated abiotic stress tolerance. In Salicylic Acid (pp. 183-247). Springer Netherlands.

20. Potters, G., Horemans, N., Jansen, M. A. K., (2010) The cellular redox state in plant stress biology - A charging concept. Plant Physiol. Bioch. 48, 292-300.

21. Sappl, P. G., Onate-Sanchez, L., Singh, K. B., Millar, A. H. (2004) Proteomic analysis of glutathione S-transferases of Arabidopsis thaliana reveals differential salicylic acid-induced expression of the plant-specific phi and tau classes. Plant Mol. Biol. 54, 205-219.

22. Sappl, P. G., Carroll, A. J., Clifton, R., Lister, R., Whelan, J., Millar, A. H., Singh, K. B. (2009) The Arabidopsis glutathione transferase gene family displays complex stress regulation and co-silencing multiple genes results in altered metabolic sensitivity to oxidative stress. Plant J. 58, 53-68.

23. Schafer, F. O., Buettner, G. R. (2001) Redox environment of the cell as viewed through the redox state of glutathione disulfide/glutathione couple. Free Radic. Biol. Med. 30, 11911212.

24. Soltész, A., Tímár, I., Vashegyi, I., Tóth, B., Kellős, T., Szalai, G., Vágújfalvi, A., Kocsy, G., Galiba, G. (2011) Redox changes during cold acclimation affect freezing tolerance but not the vegetative/reproductive transition of the shoot apex in wheat. Plant Biol. 13, 757-766. 
25. Szalai, G., Kellős, T., Galiba, G., Kocsy, G. (2009) Glutathione as an antioxidant and a regulatory molecule in plants under abiotic stress conditions. J. Plant Growth. Regul. 28, 6680.

26. Tari, I., Csiszár, J., Horváth, E., Poór, P., Takács, Z., Szepesi, Á. (2015) Alleviation of the adverse effect of salt stress in tomato by salicylic acid shows time- and organ-specific antioxidant response. Acta Biol. Cracov. Bot. (In press)

27. Tolin, S., Arrigoni, G., Trentin, A. R., Veljovic-Jovanovic, S., Pivato, M., Zechman, B., Masi, A. (2013) Biochemical and quantitative proteomics investigations in Arabidopsis ggt1 mutant leaves reveal a role for the gamma-glutamyl cycle in plant's adaptation to environment. Proteomics 13: 2031-2045.

28. Wagner, U., Edwards, R., Dixon, D. P., Mauch, F. (2002) Probing the diversity of the Arabidopsis glutathione S-transferase gene family. Plant Mol. Biol. 49, 515-532. 
Figure legends

Fig. 1 Cell viability in leaves and root tips of Wt (Col-Wt) and Atgstf9 Arabidopsis treated with different $\mathrm{NaCl}(50 \mathrm{mM}$ or $150 \mathrm{mM})$ or $\mathrm{SA}\left(10^{-7} \mathrm{M}\right.$ or $\left.10^{-5} \mathrm{M}\right)$ concentrations. Means denoted by different letters indicate a significant difference between the treatments at a level $\mathrm{P}<0.05$ according to Duncan's test.

Fig. $2 \mathrm{H}_{2} \mathrm{O}_{2}$ and malondialdehyde (MDA) contents of Wt (Col-Wt) and Atgstf9 Arabidopsis seedlings treated with different $\mathrm{NaCl}(50 \mathrm{mM}$ or $150 \mathrm{mM})$ or $\mathrm{SA}\left(10^{-7} \mathrm{M}\right.$ or $\left.10^{-5} \mathrm{M}\right)$ concentrations. Means denoted by different letters indicate a significant difference between the treatments at a level $\mathrm{P}<0.05$ according to Duncan's test.

Fig. 3 Glutathione transferase (GST) and glutathione peroxidase (GPOX) activities of Wt (ColWt) and Atgstf9 Arabidopsis seedlings treated with different $\mathrm{NaCl}(50 \mathrm{mM}$ or $150 \mathrm{mM})$ or SA $\left(10^{-7} \mathrm{M}\right.$ or $\left.10^{-5} \mathrm{M}\right)$ concentrations. Means denoted by different letters indicate a significant difference between the treatments at a level $\mathrm{P}<0.05$ according to Duncan's test.

Fig. 4 Total, reduced and oxidized ascorbate (AsA and DHA) and glutathione (GSH and GSSG) contents of Wt (Col-Wt) and Atgstf9 Arabidopsis seedlings treated with different $\mathrm{NaCl}$ (50 mM or $150 \mathrm{mM})$ or SA $\left(10^{-7} \mathrm{M}\right.$ or $\left.10^{-5} \mathrm{M}\right)$ concentrations. Means $\pm \mathrm{SD}$. Means denoted by different letters indicate a significant difference between the treatments at a level $\mathrm{P}<0.05$ according to Duncan's test (Italic for AsA and GSH, normal letters for DHA and GSSG). * n.s. - not significant. There were no significant differences among GSSG contents. 
Table 1 Half-cell reduction potential of the GSH/GSSG redox couple ( $\mathrm{E}_{\mathrm{GSSG} / 2 \mathrm{GSH}}$ ) in Arabidopsis seedlings following $48 \mathrm{~h}$ of $\mathrm{NaCl}$ or SA treatment

\begin{tabular}{lll}
\hline & \multicolumn{2}{l}{$\mathrm{E}_{\mathrm{GSSG} / 2 \mathrm{GSH}}(\mathrm{mV})$} \\
& $\mathrm{Col}-\mathrm{Wt}$ & Atgstf9 \\
\hline Control & -267.9 & -259.5 \\
$50 \mathrm{mM} \mathrm{NaCl}$ & -239.7 & -246.9 \\
$150 \mathrm{mM} \mathrm{NaCl}$ & -254.8 & -265.3 \\
$10^{-7} \mathrm{M} \mathrm{SA}$ & -264.5 & -264.2 \\
$10^{-5} \mathrm{M} \mathrm{SA}$ & -266.5 & -264.5 \\
\hline
\end{tabular}

